\title{
Tissue-specific circadian clocks in plants
}

\author{
Author: Motomu Endo
}

Affiliations:

1. Division of Integrated Life Science, Graduate School of Biostudies, Kyoto University, Sakyo, Kyoto 606-8501, Japan.

2. Japan Science and Technology Agency, PRESTO, 4-1-8 Honcho Kawaguchi, Saitama 332-0012, Japan.

\begin{abstract}
Circadian clocks affect a large proportion of differentially expressed genes in many organisms. Tissue-specific hierarchies in circadian networks in mammals have been contentiously debated, whereas little attention has been devoted to the concept in plants, owing to technical difficulties. Recently, several studies have demonstrated tissue-specific circadian clocks and their coupling in plants, suggesting that plants possess a hierarchical network of circadian clocks. The following review summarizes recent studies describing the tissue-specific functions and properties of these circadian clocks and discusses the network structure and potential messengers that might share temporal information on such a network.
\end{abstract}

\section{Introduction}

The rotation and orbit of the earth bring daily light/dark rhythms and seasonal rhythms. To anticipate and cope with recurring environmental changes, many organisms have developed a circadian clock, which increases their fitness by regulating behavior and gene expression. In many multicellular organisms, more than a dozen circadian clock genes have been identified, and most of them are expressed universally (Table 1), suggesting that circadian clocks generate self-sustaining and cell-autonomous oscillations in each cell [1,2]. However, these cellular circadian rhythms need to be integrated into a tissue or organismal level to achieve coordinated physiological responses. This has been extensively documented in mammals as hierarchical and tissue-specific functions of networked circadian clocks. The circadian clock in the suprachiasmatic nucleus (SCN) in the brain is known as a central oscillator, whereas clocks in peripheral tissues, such as liver, are termed peripheral oscillators [3]. In plants, many studies have clarified the molecular functions of circadian clock genes, such as 
CIRCADIAN CLOCK ASSOCIATED 1 (CCA1), TIMING OF CAB EXPRESSION 1 (TOC1), GIGANTEA (GI), PSEUDO RESPONSE REGULATOR (PRR) genes, and EARLY FLOWERING (ELF) genes. A circadian clock system comprised of these genes is tightly interlocked and has multi-feedback loops. Despite the important advances at the cellular level, however, only a few studies have investigated the tissue-specific functions of the circadian clock, and it is unclear whether a hierarchical and tissue-specific function of the plant clock exists.

Recently, several reports demonstrated tissue-specific dynamics and hierarchical coupling of circadian clocks in plants (Figure 1). This review focuses on the current state of knowledge regarding the properties of clocks in a specific cell type, and discusses prospective inter-tissue signaling mechanisms that synchronize clock information at the organismal level.

\section{Main text}

\section{Tissue-specific regulation of the circadian clock}

The first report that hypothesized tissue-specific regulation of the circadian clock came from an observation of stomatal opening, photosynthesis, and leaflet movement in bean plants [4]. The longer periodic timing of leaflet movements relative to the stomatal opening or photosynthesis period under free-running conditions suggested there are multiple circadian clocks in different tissues. Later, cellular observations were performed using transgenic tobacco cells expressing the cytosolic $\mathrm{Ca}^{2+}$-sensitive luminescent protein aequorin and LHCB::LUC [5], and the respective rhythms were shown to free-run with different periods. After that, more direct observations were reported in tobacco. The $\mathrm{Ca}^{2+}$ rhythmicity of cells and tissues was shown to oscillate with distinct differences in phase [6]. Similar tissue-specific oscillations of cytosolic $\mathrm{Ca}^{2+}$ were also seen in Arabidopsis. The circadian rhythms of $C A B$ gene expression are uncoupled from those of cytosolic $\mathrm{Ca}^{2+}$ in some situations, suggesting that distinct circadian clocks are located in different cell types [7].

Other clock-regulated genes have also displayed tissue-specific rhythmicities. The $C A B$ expression rhythm is distinct from the CHS or $P H Y B$ gene expression rhythms in free-running periods under constant light condition [8,9]. In addition, sensitivity to environmental cues is distinctly regulated by two circadian oscillators regulating CAB2::LUC and CAT3::LUC. The circadian oscillator regulating CAB2 expression responds preferentially to light/dark cycles rather than temperature cycles, whereas the circadian oscillator regulating CAT3 expression responds to temperature cycles rather than light/dark 
cycles [10]. Since CAB2 is mainly expressed in the mesophyll, but CAT3 is expressed both in mesophyll and epidermis, multiple circadian clock systems in plants have been suggested.

Clock gene expression profiles representing the core loop of the circadian clock system in Arabidopsis are also distinct in different tissues. CCA1 oscillations were shown to have a longer period and lower amplitude in guard cells [11], and had a different period in the center of the leaf than in the center of the rosette [12]. The phase of TOC1 oscillation was also distinct in the vasculature $[13,14]$. More interestingly, CCA1 displayed striped expression patterns in root tissues [15].

\section{Spatial expression patterns of clock genes}

Tissue-specific differential expression of clock genes would be the easiest way to explain such tissue-specific oscillations. Although the majority of clock genes are expressed in most cell types [16-19], some clock genes, including PRR3, PRR9, and GI, show vasculature-enriched expression patterns, based on GUS staining assays [16,20] (Table 1). The non-uniform expression of at least several clock genes among different cell types suggests that tissue-specific clock functions can be partially explained by spatial gene expression patterns.

There is continuing debate over whether these genes are exclusively expressed in vasculature; for example, GFP fluorescence from PRR3::PRR3-GFP can be detected even in mesophyll cells [21], and another transgenic line, GI::GI-GUS, suggests that GI is expressed both in mesophyll and vasculature [22]. There is a caveat to the experiments with GUS staining, however. Even if the GUS expression levels are almost the same in each tissue, apparent GUS staining levels will be largely dependent on the cell size and cell density. Since mesophyll and epidermal cells are larger, whereas vascular cells are small and tightly aligned, we supposed that the GUS staining level in the vasculature would tend to be higher. To support our view, GUS staining was more intense in the vasculature, even in 35S::GUS or equivalent transgenic lines [23,24].

To more reliably estimate clock gene expression levels in a specific tissue, a direct tissue-isolation technique with high spatiotemporal resolution was recently developed [13]. The tissue isolation and subsequent microarray analysis showed that only ELF4 was a particularly vasculature-rich gene (10 fold higher in vasculature), whereas the other clock genes, including PRR3, PRR9 and GI, were expressed both in mesophyll and vasculature, and 
PRR9 was rather a mesophyll-rich gene [13]. While the ELF4 expression pattern strongly implies that there are discrete functions of the circadian clock in vasculature, it is also likely that tissue-specific phase, period, and amplitude regulation are crucial; indeed, as evidenced by the phenotype of an elf4 mutant, not all tissue-specific oscillations can be explained by ELF4 alone. [25]. Vasculature-specific functions of cry2, COP1, and SPA1, all of which are involved in circadian clock input, might be crucial to determine the tissue specificities of the clock [26-29].

\section{Hierarchical coupling of tissue-specific clocks}

To achieve coordinate responses at an organismal level, it appears that the circadian clocks in different tissues need to be coupled with each other. In Arabidopsis cotyledons, hierarchical coupling of clocks between vasculature and mesophyll was recently reported [13]. Overexpression of CCA1 driven by a vasculature-specific SUC2 promoter caused circadian rhythm perturbation not only in vasculature but also in mesophyll cells. On the other hand, CCA1 driven by a mesophyll-specific promoter, $C A B$, disrupted circadian rhythms only in mesophyll, while robust circadian oscillation in vasculature was still observed. Similarly, the short period (18 hour) phenotype of a light-regulated WD 1 (lwd1); lwd2 double mutant was lengthened (21 hour) by SUC2-driven LWD1, suggesting that partial complementation of LWD function in vasculature affects mesophyll circadian rhythms (about 80\% of leaf cells are mesophyll) [30]. Furthermore, it has been suggested that a circadian clock in roots consists of limited numbers of components, and the expression of root clock genes is coupled to the hierarchical dominant shoot clock rhythm [31,32]. These results demonstrate that there is an asymmetric coupling of circadian clocks from vasculature to mesophyll cells and shoot (shoot vasculature?) to root, suggesting plants have a hierarchical circadian clock system.

If there is a hierarchical organization, then do plants have a central oscillator that governs a centralized network analogous to the SCN in mammal brains (Figure 2) [33]? Since food and water anticipatory behaviors are SCN independent [34], the SCN is not an absolute center for time measurement in a strict sense; however, in practice, the SCN oscillator governs the greater part of circadian responses. In that sense, animals have a more centralized clock network. Another type of network is distributed, or "hierarchy-less". In a distributed network, a circadian clock in each cell makes an equal contribution to the clock system, and will be minimally affected by the disruption of a hub. Since we have observed hierarchical coupling in the circadian clock network and clear phenotypes caused by perturbation of specific tissues, plants do not appear to have a distributed clock network. A third type of network structure is 
decentralized. In a decentralized network, tissues operate with local circadian rhythms or time measurement to accomplish coordinated responses, and the clocks in each tissue are loosely coupled to each other.

Recently, a circadian clock in shoot apex was reported to display homogenous synchronicity and therefore was proposed as a plant master clock [32]. However, since shoot apical meristem-specific perturbation of clock functions did not affect circadian clock regulated flowering [13], and there are at least two uncoupled clocks that have different sensitivities to temperature [10], it is likely that there are other hubs of circadian clocks, consistent with the decentralized network model.

\section{Candidate inter- and intra-tissue coupling factor}

In both the centralized or decentralized models, plant tissues need to share their time information with each other. However these inter-tissue and intra-tissue couplings might not be strongly coupled. Thain et al. entrained each cotyledon of a tobacco seedling with light-dark cycles or inverted dark-light cycles, respectively, and showed that opposite CAB2::LUC or PHYB::LUC oscillation remained in antiphase under continuous light conditions, suggesting that their clocks were not effectively coupled to each other [35]. We suppose that the reason for the lack of global coupling could be explained by the orthostichy of the vasculature. By contrast, two studies have clearly shown that there is weak, local coupling $[12,36]$.

How do clocks in specific tissues transmit time information to other tissues, and what are the coupling factors? Animals, and mammals especially, use neural and humoral signals to share the time information from the SCN. Presumably, in plants an analogous vascular system mediated inter-tissue "messenger" promotes time information integration. One possible candidate is sugar concentration oscillation. Sugar from photosynthesis is transported to all plant cells through the vascular system, and endogenous sugar is crucial for maintaining the amplitude of the circadian clock [37]. Consistent with this notion, the simplified root clock is synchronized by a photosynthesis-related signal from the shoot [31]. Micro RNA (miRNA) is another possible candidate for inter- and intra-tissue coupling. For example, in root growth or phosphate homeostasis, short distance and long distance miRNA transport through plasmodesmata and the vascular system were reported [38,39]. The presence of tissue-specifically expressed miRNAs lends support for this hypothesis [40]. At present, there is no iron-clad evidence that miRNA directly affects the plant circadian clock system; but in 
mammals or insects, miRNA has a significant role in clock regulation [41].

\section{Concluding remarks}

Apart from the extensive characterization of genetic circuits implicated in the 'averaged' circadian clock system at a whole-plant level, only a few attempts have so far been made at analyzing tissue-specific clock and inter-/intra-tissue time information sharing. As illustrated above, plants have a hierarchical circadian clock system somewhat like animals. To better understand the coupling mechanisms and the features of clocks in each tissue, one should not only measure gene expression levels, but also focus on post-transcriptional and post-translational regulation in a specific tissue. From a practical perspective, one key objective is the application to crop plants of any forthcoming molecular insights into the tissue-specific circadian clock system. Some clock genes underlie quantitative trait loci that will improve agricultural traits such as flowering, yield, biomass, disease resistance, and so on [42]. Modification of a circadian clock system in a specific tissue might allow us to manipulate specific agricultural traits regulated by the targeted tissue. As plants and flies have tissue-specific cis regulatory signatures [13, 43], an initial approach might combine Chip-seq and high-throughput transcription factor screening to identify key molecules that determine distinct circadian clock systems in specific tissues.

Presently, we still have only a limited number of tools for the tissue-specific analysis of circadian clocks, although we have established a few techniques, such as rapid tissue-isolation, Tissue-Specific Luciferase Assay (TSLA), and tissue-specific disruption of clock functions [13]. In addition to the improvement of these techniques, microscopic observations with an unstable variant of EGFP (e.g., d2EGFP), differentiation induction techniques to obtain single cell types, or laser capture micro dissection will create new avenues to investigate circadian clocks at a tissue or cell level.

\section{Acknowledgements}

I thank James Alan Hejna of the Kyoto University for his critical reading and contributions to the manuscript. This work was supported by a JST PRESTO 888067, JSPS KAKENHI grants 22770036, 25650097 and 15H05958, a Nakatani Foundation, and a Mitsubishi Foundation. 
Table 1. Spatial expression patterns of circadian clock genes

\begin{tabular}{|c|c|c|c|c|}
\hline \multirow{2}{*}{ Clock genes } & \multicolumn{4}{|c|}{ Spatial expression patterns determined by } \\
\hline & GUS staining & LUC assay & Transcr & tome analysis [13] \\
\hline TOC1 & Whole seedling [16] & Whole seedling [13] & & Slightly vasculature rich \\
\hline CCA1/LHY & Whole seedling [17] & Whole seedling [14] & & Slightly mesophyll rich \\
\hline PRR3 & Vasculature rich [16] & & & \\
\hline$P R R 7$ & & & At least in both & Mesophyll rich \\
\hline PRR9 & Vasculature rich [16] & & vasculature & Mesophyll rich \\
\hline ELF4 & & & & Vasculature rich \\
\hline$G I$ & $\begin{array}{l}\text { Whole or vascular rich } \\
{[20,22]}\end{array}$ & & & \\
\hline The others & & & & \\
\hline
\end{tabular}

\section{Figure legends}

\section{Figure 1}

Tissue-specific circadian clocks and their communications. Red arrows indicate inter- and intra-organ/tissue communications. (a) There is a simplified clock in roots, and the clock is regulated by a shoot clock through photosynthate(s) (inter-organ asymmetric coupling) [35]. (b) A clock in the vasculature regulates the mesophyll clock, whereas the mesophyll clock scarcely affects the vasculature clock (inter-tissue asymmetric coupling) [15]. (c) Mesophyll cells in a leaf are weakly coupled and produce spiral waves (intra-organ coupling) [14, 41]. In contrast to these coupling mechanisms, locally anti-phased circadian rhythms are not synchronized to the clocks in the other leaf (d), or in the other part of the same leaf (dashed arrows) (e) [38]. Furthermore, several clocks in a specific type of cell show distinct circadian rhythms. (f) Circadian rhythms in guard cells have a longer period and lower amplitude [13]. (g) Mesophyll and epidermal clocks might have distinct sensitivities to light and temperature signals [12]. (h) Circadian oscillation of clock gene expression has a striped pattern along with root growth [17].

\section{Figure 2}

Schematic models of centralized, decentralized, and distributed networks. Squares indicate a responsive cell/tissue/organ that functions as a hub. Circles indicate targeted cells that operate specific circadian responses such as gene expression, redox regulation, cell elongation, flowering, and so on. In terms of efficiency, a centralized network has a higher performance, but is less robust than a distributed network. A centralized network can be destroyed by perturbation of a small number of hubs. As the mammalian SCN is the center of circadian rhythms in most cases, the mammalian circadian clock is generally assumed to be a 
centralized network. The observed hierarchical coupling of clocks in plants suggests that the plant circadian clock network should be centralized or decentralized, because a distributed network is known to be a hierarchy-less network.

\section{References and recommended reading}

Papers of particular interest, have been highlighted as:

- of special interest

•• of outstanding interest

1. Doherty CJ, Kay SA: Circadian control of global gene expression patterns. Annu Rev Genet. 2010, 44:419-444.

2. Jolma IW, Laerum OD, Lillo C, Ruoff P: Circadian oscillators in eukaryotes. Wiley Interdiscip Rev Syst Biol Med. 2010, 2:533-549.

3. Barclay JL, Tsang $\mathrm{AH}$, Oster $\mathrm{H}$ : Interaction of central and peripheral clocks in physiological regulation. Prog Brain Res. 2012,199:163-181.

4. Hennessey TL, Field CB: Evidence of multiple circadian oscillators in bean plants. $J$ Biol Rhythms. 1992, 7:105-113.

5. Sai J, Johnson CH: Different circadian oscillators control $\mathrm{Ca}^{2+}$ fluxes and Lhcb gene expression. Proc Natl Acad Sci USA. 1999, 96:11659-11663.

6. Wood NT, Haley A, Viry-Moussaid M, Johnson CH, van der Luit AH, Trewavas AJ: The calcium rhythms of different cell types oscillate with different circadian phases. Plant Physiol. 2001, 125:787-796.

7. Xu X, Hotta CT, Dodd AN, Love J, Sharrock R, Lee YW, Xie Q, Johnson CH, Webb AA: Distinct light and clock modulation of cytosolic free $\mathrm{Ca}^{2+}$ oscillations and rhythmic CHLOROPHYLL A/B BINDING PROTEIN2 promoter activity in Arabidopsis. Plant Cell. 2007, 19:3474-3490.

8. Thain SC, Murtas G, Lynn JR, McGrath RB, Millar AJ: The circadian clock that controls gene expression in Arabidopsis is tissue specific. Plant Physiol. 2002 130:102-110.

9. Hall A, Kozma-Bognar L, Bastow RM, Nagy F, Millar AJ: Distinct regulation of CAB and PHYB gene expression by similar circadian clocks. Plant J. 2002, 32:529-537.

10. Michael TP, Salome PA, McClung CR: Two Arabidopsis circadian oscillators can be distinguished by differential temperature sensitivity. Proc Natl Acad Sci USA. 2003, 100:6878-6883.

-• In this study, the authors demonstrate that two different circadian clocks can be 
distinguishable by their sensitivity to the light-dark cycle and warm-cold cycle, suggesting tissue-specific circadian clocks have distinct roles in processing environmental signals.

11. Yakir E, Hassidim M, Melamed-Book N, Hilman D, Kron I, Green RM: Cell autonomous and cell-type specific circadian rhythms in Arabidopsis. Plant J. 2011, 68:520-531.

12. Fukuda H, Nakamichi N, Hisatsune M, Murase H, Mizuno T: Synchronization of plant circadian oscillators with a phase delay effect of the vein network. Phys Rev Lett. 2007, 99:098102.

13. Endo M, Shimizu H, Nohales MA, Araki T, Kay SA: Tissue-specific clocks in Arabidopsis show asymmetric coupling. Nature 2014, 515:419-422.

14. Shimizu H, Araki T, Endo M: Photoperiod sensitivity of the Arabidopsis circadian clock is tissue-specific. Plant Signal Behav. 2015, 10:e1010933.

15. Fukuda H, Ukai K, Oyama T: Self-arrangement of cellular circadian rhythms through phase-resetting in plant roots. Phys Rev E Stat Nonlin Soft Matter Phys. 2012, 86:041917.

- The authors demonstrate that the root clock exhibits stripe waves of gene expression originating at the root tip. This phenomenon results from a continuous phase resetting of circadian oscillations there.

16. Para A, Farré EM, Imaizumi T, Pruneda-Paz JL, Harmon FG, Kay SA: PRR3 Is a vascular regulator of TOC1 stability in the Arabidopsis circadian clock. Plant Cell. 2007,19:3462-3473.

- The authors revealed that PRR3 and PRR9 show vascular-enriched expressions, at least in GUS assays. They provide an important concept that there should be a tissue-specific regulation of the circadian clock, even in plant leaves.

17. Pruneda-Paz JL, Breton G, Para A, Kay SA: A functional genomics approach reveals CHE as a component of the Arabidopsis circadian clock. Science. 2009, 323:1481-1485.

18. Chow BY, Sanchez SE, Breton G, Pruneda-Paz JL, Krogan NT, Kay SA: Transcriptional regulation of LUX by CBF1 mediates cold input to the circadian clock in Arabidopsis. Curr Biol. 2014, 24:1518-1524.

19. Xie Q, Wang P, Liu X, Yuan L, Wang L, Zhang C, Li Y, Xing H, Zhi L, Yue Z et al.: LNK1 and LNK2 are transcriptional coactivators in the Arabidopsis circadian oscillator. Plant Cell. 2014, 26:2843-2857.

20. Edwards J, Martin AP, Andriunas F, Offler CE, Patrick JW, McCurdy DW: GIGANTEA is a component of a regulatory pathway determining wall ingrowth deposition in phloem parenchyma transfer cells of Arabidopsis thaliana. Plant J. 2010, 63:651-661.

21. Fujiwara S, Wang L, Han L, Suh SS, Salomé PA, McClung CR, Somers DE: 
Post-translational regulation of the Arabidopsis circadian clock through selective proteolysis and phosphorylation of pseudo-response regulator proteins. $\mathrm{J}$ Biol Chem. 2008, 283:23073-23083.

22. Koo J, Kim Y, Kim J, Yeom M, Lee IC, Nam HG: A GUS/luciferase fusion reporter for plant gene trapping and for assay of promoter activity with luciferin-dependent control of the reporter protein stability. Plant Cell Physiol. 2007, 48:1121-1131.

23. Sheldon CC, Hills MJ, Lister C, Dean C, Dennis ES, Peacock WJ: Resetting of FLOWERING LOCUS C expression after epigenetic repression by vernalization. Proc Natl Acad Sci USA. 2008, 105:2214-2229.

24. Marrocco K, Thomann A, Parmentier Y, Genschik P, Criqui MC: The APC/C E3 ligase remains active in most post-mitotic Arabidopsis cells and is required for proper vasculature development and organization. Development 2009, 136:1475-1485.

25. Doyle MR, Davis SJ, Bastow RM, McWatters HG, Kozma-Bognár L, Nagy F, Millar AJ, Amasino RM: The ELF4 gene controls circadian rhythms and flowering time in Arabidopsis thaliana. Nature 2002, 419:74-77.

26. Endo M, Mochizuki N, Suzuki T, Nagatani A: CRYPTOCHROME2 in vascular bundles regulates flowering in Arabidopsis. Plant Cell 2007, 19:84-93.

27. Ranjan A, Fiene G, Fackendahl P, Hoecker U: The Arabidopsis repressor of light signaling SPA1 acts in the phloem to regulate seedling de-etiolation, leaf expansion and flowering time. Development 2011, 138:1851-1862.

- This study demonstrated that SPA1 functions specifically in vasculature. The results indicate that there are tissue-specific regulation mechanisms not only in circadian clock but also in light signaling.

28. Jang S, Marchal V, Panigrahi KC, Wenkel S, Soppe W, Deng XW, Valverde F, Coupland G: Arabidopsis COP1 shapes the temporal pattern of $\mathrm{CO}$ accumulation conferring a photoperiodic flowering response. EMBO J. 2008, 27:1277-1288.

29. Wang H, Wang $\mathrm{H}$ : Multifaceted roles of FHY3 and FAR1 in light signaling and beyond. Trends Plant Sci. 2015, 20:453-461.

30. Wang PH, Hsu CR: Do Clocks in Different Arabidopsis Tissues Communicate with Each Other? 2013, http://proj1.sinica.edu.tw/ hispj/news/paper/101/9902399038.pdf

31. James AB, Monreal JA, Nimmo GA, Kelly CL, Herzyk P, Jenkins GI, Nimmo HG: The circadian clock in Arabidopsis roots is a simplified slave version of the clock in shoots. Science 2008, 322:1832-1835.

•- In this study, the authors demonstrate that there is a simplified clock in the roots compared to the shoots. They also revealed that sucrose from the shoots affects circadian rhythms of the 
roots. This is the first report that there is a long distance communication for time information sharing between different tissues or organs.

32. Takahashi N, Hirata Y, Aihara K, Mas P: A Hierarchical Multi-oscillator Network Orchestrates the Arabidopsis Circadian System. Cell 2015, 163:148-159.

•- In this study, the authors demonstrate that a clock in the shoot apex is more synchronized than the other tissue/organs tested, probably due to intra-organ (or tissue) coupling. They also showed that a clock in the shoot apex affects circadian oscillation in root tissues.

33. Baran P: On Distributed Communications: I. Introduction to Distributed Communications Networks. http://www.rand.org/publications/RM/baran.list.html., RM-3420-PR.

34. Tahara Y, Shibata S: Chronobiology and nutrition. Neuroscience 2013, 253:78-88.

35. Thain SC, Hall A, Millar AJ: Functional independence of circadian clocks that regulate plant gene expression. Curr Biol. 2000, 10:951-956.

•• This paper concludes that circadian clocks in each cell do not have tight coupling. Two cotyledons that have anti-phase rhythms did not affect each other. The same can be seen even in the same cotyledon.

36. Wenden B, Toner DL, Hodge SK, Grima R, Millar AJ: Spontaneous spatiotemporal waves of gene expression from biological clocks in the leaf. Proc Natl Acad Sci USA. 2012, 109:6757-6762.

37. Haydon MJ, Mielczarek O, Robertson FC, Hubbard KE, Webb AA: Photosynthetic entrainment of the Arabidopsis thaliana circadian clock. Nature 2013, 502:689-692.

38. Sevilem I, Miyashima S, Helariutta Y: Cell-to-cell communication via plasmodesmata in vascular plants. Cell Adh Migr. 2013, 7:27-32.

39. Pant BD, Buhtz A, Kehr J, Scheible WR: MicroRNA399 is a long-distance signal for the regulation of plant phosphate homeostasis. Plant J. 2008, 53:731-738.

40. Breakfield NW, Corcoran DL, Petricka JJ, Shen J, Sae-Seaw J, Rubio-Somoza I, Weigel D, Ohler U, Benfey PN: High-resolution experimental and computational profiling of tissue-specific known and novel miRNAs in Arabidopsis. Genome Res. 2012, 22:163-176. 41. Mehta N, Cheng HY: Micro-managing the circadian clock: The role of microRNAs in biological timekeeping. $J$ Mol Biol. 2013, 425:3609-3624.

42. Bendix C, Marshall CM, Harmon FG: Circadian Clock Genes Universally Control Key Agricultural Traits. Mol Plant. 2015, pii: S1674-2052(15)00171-9.

43. Meireles-Filho AC, Bardet AF, Yáñez-Cuna JO, Stampfel G, Stark A: cis-regulatory requirements for tissue-specific programs of the circadian clock. Curr Biol. 2014, 24:1-10. 
(c) ( ) (e)

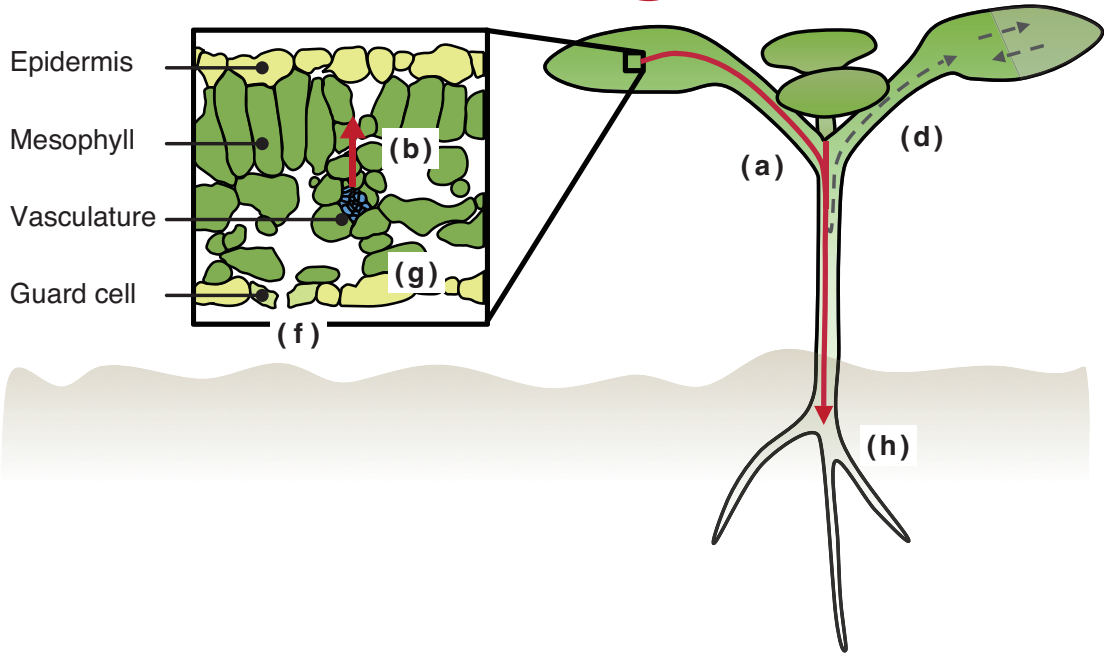

Inter- and intra-organ/tissue coupling

(a) Shoot and/or shoot apex $\rightarrow$ Root

(b) Vasculature $\rightleftarrows$ Mesophyll

(c) Spiral wave in a leaf

No inter- and intra-organ coupling

(d) A leaf to the other side of a leaf

(e) Between tip and bottom of leaf

\section{Distinct circadian clocks}

( f ) Guard cell vs. Epidermis, Mesophyll

(g) Mesophyll vs. Epidermis (?)

(h) Stripe wave in a root 
